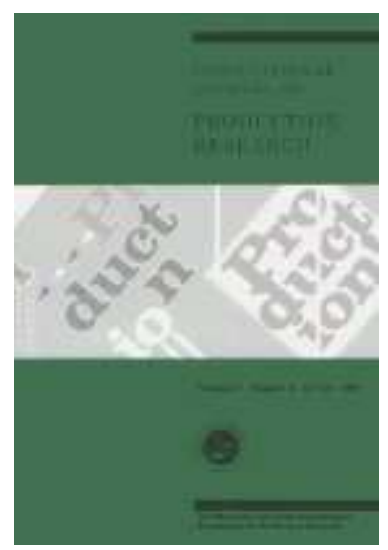

\title{
Make to stock and mix to order: Choosing intermediate products in the food-processing industry
}

\begin{tabular}{|r|l|}
\hline Journal: & International Journal of Production Research \\
\hline Manuscript ID: & TPRS-2008-IJPR-0783.R2 \\
\hline Manuscript Type: & Original Manuscript \\
\hline $\begin{array}{r}\text { Date Submitted by the } \\
\text { Author: }\end{array}$ & 12-Jan-2009 \\
\hline & $\begin{array}{l}\text { Complete List of Authors: } \\
\text { Management Engineering } \\
\text { and Business } \\
\text { Van Donk, Dirk Pieter; University of Groningen, Faculty of } \\
\text { Economics and Business }\end{array}$ \\
\hline Keywords: & POSTPONEMENT, OPERATIONS MANAGEMENT \\
\hline Keywords (user): & food industry, decoupling point \\
\hline
\end{tabular}

\section{scholarONE \\ Manuscript Central}




\begin{abstract}
In contrast to discrete manufacturers, food-processing companies can sometimes produce the same end products in different ways: either mix first and then process, or process first and mix later. Moreover, a final product can be mixed from different raw materials or intermediates. That adds a new dimension to postponement and decoupling point theory as choices have to be made not only with regard to where to locate inventory, but also which products to store. That aspect has not been covered so far. This paper explores this problem for a typical two-stage food production situation in a flour mill. The number and composition of intermediate products in the decoupling point is determined using a stepwise solution approach supported by mathematical programming models. The procedure facilitates decision-making for the management of the mill regarding how many and what intermediates to store. Extensions of the models presented might be helpful to solve related problems such as determining the number of intermediate storage tanks required.
\end{abstract}

Keywords: food industry, decoupling point, postponement, intermediate storage, case study

\title{
1. Introduction
}

In the food processing industry, a typical production plant produces a multitude of intermediate products in an even wider range of packages. Often, the output variety is based on a relatively small number of (agricultural) raw materials. In general, such a divergent product structure is typical for the process industries (Fransoo and Rutten, 1994). Differences in products can be associated with customer-specific products, either in packaging form, size, or print, labelling, or (more fundamental) product recipe. Recipes normally differ with respect to a bundle of product quality attributes. Often a recipe can be characterised in terms of minimal requirements for each attribute. Different options might exist in relation to where in the process a recipe is made specific: either adding specific ingredients early or late in the process.

A number of factors and trade-offs determine at what stage a product will become customerspecific. Generally, product specifications are assumed to be known for each stage of the production process. In other words, the final product determines the requirements and specification of all intermediates univocally. However, in food-processing companies - specifically those that mix and blend - recipes of final products can sometimes be manufactured in different ways. Two extreme possibilities are either starting with a customer-specific recipe and process the resulting relatively small batches and store final product waiting for orders, or process raw materials in large batches, store these and mix them to recipe as the last step as customer orders arrive. It might be clear that 
there is an endless number of variants in between in terms of the amount and nature of possible intermediate recipes that can be stored, and deciding how to organize this is an essential factor in determining the operational performance of many mix or blend production systems. Finally, given the nature of recipes, one might decide to deliver products having a too high quality for one or two requirements. In general, each of the alternatives has implications for the processing costs, but, additionally, it might also affect the costs of the raw materials used. This problem has some similarities with binning, where quality grades are determined and subsequently downgrade possibilities are used to satisfy demand with higher quality levels to save setups (see Lyon et al., 2001). However, their problem is operational (how to satisfy orders), whereas we focus on a tactical decision (what are the recipes for a certain period of time). Moreover, in discrete manufacturing far less possibilities exist for the product structure (recipe).

So far, the literature has not addressed the problem of jointly determining $(i)$ when to specify a product to customer specifications, $(i i)$ which recipes to use for flexible products -i.e., intermediate products that can still be used to mix various customer orders, and (iii) how many intermediate products to use. This paper develops an approach to decide on the number and composition of intermediate products in two-stage food production systems, supported by quantitative modelling. More specifically, we consider the case of a flour mill, facing the problem of how much and which intermediate recipes to store and to determine the consequences of different options. These consequences range from operational issues such as the costs of ingredients to more tactical and strategic decisions such as the required number of intermediate silos. More generally, the aim of this paper is also to contribute to the understanding of product specification in the food industry if end products can either be made to stock, mixed to order, or made to order.

The paper is structured as follows. In the next section, the theoretical background of the studied problem is discussed. Then, the case study is introduced, also outlining the quality parameters of the products in question. The following section then presents the solution procedure for determining the number and composition of the intermediate products. Subsequently, the results of the application of the model in the case study are discussed. Finally, the last section will present our conclusions and a discussion on the paper's contribution and the future research opportunities.

\section{Theoretical background}

Resulting from increasingly powerful retailers (Dobson et al., 2001), food manufacturers find themselves in a situation where the downstream supply chain requires more and more flexibility, whereas the characteristics of the production systems involved often do not support this (Van Donk et al., 2008). To improve their flexibility, while still trying to produce in efficient volumes, food manufacturers often try to postpone the diverging of their product mix as long as possible (e.g., Van Donk, 2001; Soman et al., 2004). This late specification allows for shorter lead times, while keeping production efficiency before the specification on an acceptable level. Early product specification would lead to an early diverging of the number of different products and would result in increased requirement of storage facilities (as each product requires its own tank or silo), and numerous additional changeovers and/or cleaning activities.

This concept of 'postponement' (or delayed differentiation) has been extensively studied in the literature. In manufacturing situations, postponement aims to retain products in a neutral and noncommitted status as long as possible (Yang et al., 2004b). An overview of the research on postponement can be found in Van Hoek (2001), which was updated and extended by Boone et al. (2007). They propose several new challenges for further research. Among others, they conclude that the application of postponement was not as widespread as was expected based on the attention in the literature. Recently, Forza et al. (2008) developed a typology that identifies three types of postponement. They stress that it is important to clarify which type is studied, to be able to carefully assess the resulting operational performance. The first type they identify deals with postponement of product specification from the forecast-driven production stages to the order-driven production stages. In our work, when deciding on when to specify products, and what flexible recipes to use to mix final products, this is the kind of situation we are dealing with. However, the specific role of 
recipes in the product structure encountered in most process industries is not explicitly considered by Forza et al. (2008).

Next to his 2001 review, Van Hoek also wrote specifically about postponement in the food industry (Van Hoek, 1999). Here, he noted that, in comparison with other industries, the application of postponement in the food industry is fairly low. Food-specific characteristics like e.g. perishability and short lead times limit the applicability of postponement. Van Hoek however sees a strong focus on product standardization in the food industry, as this is the only way to increase capacity utilization of capital intensive production technology. The problem here is that standardization is often difficult to achieve, as customers nowadays demand more and more product variety. For this reason, Abukhader and Jonson (2007) encourage food companies to analyze their product mix and product development process in detail, to see where postponement can be applied.

However, the divergence in the product mix does not have to happen at the first production stage, but can often be placed later in the production process. Here, the concept of postponement is strongly connected to the decoupling point concept (Hoekstra and Romme, 1992; Olhager, 2003). Van Donk (2001) elaborates on this concept for the food-processing industry. One of the shortcomings he mentions is its qualitative nature and its focus on individual products as opposed to the entire product portfolio. Another issue is that the decoupling point concept assumes that specifications of products and intermediate products are known and fixed. However, as explained earlier that is often not the case in process industries and food processing.

A number of (process) industries faces the situation where once it is decided to locate the decoupling point at the intermediate product level, the set of intermediate products still has to be determined. That generally applies to the specification and/or the number of intermediates. For instance, in steel mills, an extensive range of slab lengths is demanded by customers. To reduce inventory costs (and often also to increase processing efficiency), a limited number of those slab lengths are held in storage, and are cut to customer specification when final orders arrive. Next to reducing inventory costs, this introduces waste costs due to cutting losses. In the literature we find several approaches dealing with this specific case. Recent examples are the work by Caux et al. (2006), who develop a mathematical model to evaluate the cost trade-off and the graphical approach presented by Kerkkänen (2007). A similar example can be found in the cardboard industry, where a limited number of large sheet sizes are subsequently cut into a wide variety of small sheets (Wanders et al., 2004).

In the food industry, determining the set of intermediate products mostly deals with products that are to be used in combinations to comply with the requirements on the final product (e.g. Rajaram et al., 1999). Once the intermediates are determined, a certain stock level will be maintained from which the intermediates are blended to order to create the final wide range of products. However, in theory, almost any possible set of intermediates could be used, but intermediate storage possibilities are often limited (Akkerman et al., 2007), which causes a very complex decision problem. The increasing importance of food safety and food quality (e.g., Griffith, 2006) even adds to this complexity, due to additional constraints.

The composition (or recipe) of the intermediate products has to be derived from the required specifications of the final products and the given attributes or qualities of the raw materials. As the composition of raw materials is often variable for food products, the composition of the intermediate products either has to be robust to these changes or should be changed on a regular basis. In related work by Rutten and Bertrand (1998) the concept of recipe flexibility is introduced to deal with $(i)$ variations in raw material quality, (ii) cost minimization by raw material selection, and (iii) substitutions of raw materials in recipes due to unavailability (see also Rutten, 1995). In Rutten and Bertrand (1998), only a single mixing stage is present, and determining recipes is an operational problem that can be solved for each production order. This also holds for the case of chemical fertilizers studied by Ashayeri et al. (1994). Similarly, Lyon et al. (2001) present an operational method in discrete manufacturing that matches different quality grades to orders by using higher qualities than required to prevent set-ups. However, in two-stage production systems, 
that would imply that both processes can produce order-driven, which is often not the case and certainly not in the case of flour milling we present here.

In this paper, specification (and hence order-driven production) starts at the intermediate storage stage, and the aim is to design several intermediate products that can be used in a mix-to-order strategy to fulfil future demand. This means the determination of recipes takes place not on an operational level, but on a strategic or tactical level, and is only performed once in a while to achieve cost minimization. To support this, we develop a stepwise approach supported by mathematical programming models, which takes a wide variety of typical food-related quality aspects into account. The resulting model can be used to design recipes for intermediate products, which can be used in a cost-efficient mix-to-order strategy. We feel that the approach presented in this paper will support the decision-making on postponement and the decoupling point in practice, taking into account several operational characteristics that are typical for the food industry, and addressing the inherent trade-offs between product flexibility and material costs. Furthermore, it contributes to the theory on postponement and the decoupling point, by providing a structured approach based on quantitative modelling, balancing several cost factors and including the whole product range in the decision making process.

\section{Case description}

We study a medium-sized flour manufacturer, supplying flour products to bakeries and industrial manufacturers. Figure 1 shows an outline of the production process. Grains and additional ingredients are pre-processed, blended, and milled to obtain a selection of intermediate products, which can already be blends of various grains. These products are then mixed into a wide range of flour products, transported in bulk to industrial customers and large bakeries, or packaged in bags for smaller traditional bakeries.

\section{[FIGURE 1 ABOUT HERE]}

\subsection{Problem description}

Although flour mills seem to be relatively simple processes, the above-sketched situation fully applies: a small amount of raw materials (around 10 types of grain) and an increasing amount of partly customer-specific end products (currently around 50). The two extreme options are either to mix first and then mill, or to mill all raw grains separately and mix them to recipe. Both these extremes are not reasonable due to the fact that it would either lead to a few intermediates, which would always have to be mixed, or many intermediates which would already be customer-specific. The first option would lead to a lot of mixing operations, whereas the latter situation would require a huge storage capacity. Taking this into account, the challenge is to find a solution in-between these extremes. Therefore, it is logical to store a limited number of (possibly blended) intermediate products and use these to mix end products. In terms of the decoupling point, we can characterize this as mix to order. It is worth stressing that some intermediates can be used directly as end products without mixing and after mixing in other products. An important reason for the mix-toorder strategy is the required short delivery time. Operating this system incurs all types of operational problems. Here, we address a more tactical oriented design problem: determining the amount and composition of intermediate products. On the one hand a low number of intermediates will increase the efficiency and quality of the milling operation by enabling milling in larger batches, as well as limiting the number of intermediate storage silos. On the other hand, a low number has two downsides. First, to be able to mix final products from a lower number of intermediate products, more expensive, high-quality raw materials have to be used, leading to an increase in material costs. Secondly, to mix from a smaller number the wide variety of end products, more mixing operations would be necessary, increasing operational cost of mixing.

Now, the main problem is to determine the number and composition of intermediate products, increasing operational efficiency, product quality, and flexibility on the one hand, while on the 
other hand keeping material costs and operational costs from exploding. Currently, this design is based upon intuition, craftsmanship, and habit, which are not supported by an evaluation of costs incurred. Currently, management envisages an increase in volume, which will mainly be realized by adding customer specific recipes, but is afraid that more intermediate products will be needed. The current project aims at benchmarking current practice, developing a sound understanding of the trade-off between different performance measures and costs, and to support future decisions with respect to investments in silos and mixing capacity. The aim is to develop a model to support this important design and selection decision.

\subsection{Quality parameters}

For the final products produced by the company, a variety of quality parameters are important. These parameters also need to be considered in the design of intermediate products. Wherever possible, we will not go into details on the specific details of the chemical properties or the units in which they are measured, as this would only distract from the problem discussed in this paper. There are nine quality parameters relevant in determining the intermediate products (and final products). It concerns:

\section{Protein percentage.}

2. Water absorption ability.

3. Dough extensibility, parameter 1, length of the extensometer curve.

4. Dough extensibility, parameter 2, height of the extensometer curve.

5. Deoxynivalenon (DON) level, a mycotoxin that can affect health when present at high levels.

6. A product usability index for use in bread, to cover usage parameters that are difficult to measure, assigned by the baker from the test bakery.

7. A second (similar) product usability index, this one for use in biscuits.

8. Falling number, which indicates the sprout damage of the grain used.

9. Bread volume, which is determined in a test bakery.

In creating different intermediate products or different end products, these parameters have to be considered. Most of these parameters lead to simple linear relationships in a blending process (i.e., weighted averages):

$$
Q_{p k}=\sum_{i \in I} f_{i p} Q_{i k} \quad \forall k \in\{1, \ldots, 8\}, \forall p \in P,
$$

where $f_{i p}$ is the blending fraction of raw material $i$ for intermediate product $p, Q_{p k}$ quality parameter $k$ for intermediate product $p$ (or raw material $i$ ), $I$ the set of raw materials, $P$ the set of intermediate products, and $K$ the set of quality parameters involved. Only the last (ninth) quality parameter, bread volume $\left(Q_{p, 9}\right)$, acts differently, and is calculated as follows:

$$
Q_{p, 9}=1000 \times \ln \left(\sum_{i \in I} f_{i p} e^{\frac{Q_{i, 9}}{1000}}\right) \quad \forall p \in P
$$

When relating quality parameter $Q_{p, 9}$ to minimum and maximum values $Q_{p, 9}^{\min }$ and $Q_{p, 9}^{\max }$, we would normally get a nonlinear constraint like:

$$
Q_{p, 9}^{\min } \leq 1000 \times \ln \left(\sum_{i \in I} f_{i p} e^{\frac{Q_{i, 9}}{1000}}\right) \leq Q_{p, 9}^{\max } \quad \forall p \in P
$$


For use in the MILP models in the remainder of the paper, the nonlinearity of this restriction on $f_{i p}$ is undesirable. However, such a constraint can be linearized in $f_{i p}$ by 'moving' the nonlinearity into the quality parameters, as follows:

$$
e^{\frac{Q_{p, 9}^{\min }}{1000}} \leq \sum_{i \in I} f_{i p} e^{\frac{Q_{i, 9}}{1000}} \leq e^{\frac{Q_{p, 9}^{\max }}{1000}} \quad \forall p \in P
$$

\section{Solution approach}

The main aim of the models presented in this paper is to support the current organizational decisionmaking processes. The current process basically consists of three steps: (1) consider all possible intermediates; (2) find the most economical way to make them from raw materials; and (3) select a limited number to be actually used, implicitly aiming at balancing material and mixing costs. As said, this selection process is done a few times a year. Here we aim at supporting the second and third step and leave the first one to the experience of the people involved. However, given the numerical support a wider set of intermediates can be considered. Given the restrictions, we develop two mixed-integer linear programming (MILP) models. The first one simply calculates the optimal composition of an intermediate, while the second one chooses the best intermediate if we restrict the number to be used. This stepwise approach is illustrated in Figure 2.

\section{[FIGURE 2 ABOUT HERE]}

First, a set of potential intermediate products is defined. Secondly, the optimal composition of these potential intermediate products is determined using a first MILP model. Third and finally, a selection of the potential intermediate products is made to compose the final products using a second MILP model.

Next to the correspondence with the current decision-making process, this stepwise approach also fits well with the decoupling point concept, as all three steps are centred around the decoupling point. After defining potential products to be stored at the decoupling point, one model aims to optimize the upstream process (blending and milling the intermediate products), while the second model aims to optimize the downstream process (selecting and mixing the intermediate products).

In the following paragraphs, the three stages will be further elaborated upon.

\subsection{Defining potential intermediate products}

Finding the right potential intermediate products is a difficult task. Even for relatively small-scale problems, the number of combinations is enormous. Including nine different quality parameters (as presented in Section 3) also means that care should be taken not to design potentially infeasible recipes.

What we learn from this is the need for careful consideration in the process of defining or designing intermediate products. Given the importance and difficulty, this step is executed by the experts from the recipe management and quality management department. The process of designing intermediates was supported by available recipe information (on raw materials, current intermediate products, and final products) from management information systems to validate the obtained information.

Finally, this resulted in the design of a set of over 70 possible intermediate products, covering a wide range for the quality parameters. Next to newly designed 'flexible' recipes, this set also includes recipes for final products (on customer specification), as this allows the solution approach to store this product on the intermediate storage level, thereby reducing further specification (i.e. mixing) costs. In this way, the approach can select a combination of final recipes and flexible recipes. It is worth noting that both can be used in mixing.

Initially, the model was validated with the current set of intermediate products as potential intermediate products. Allowing all of them to be used in the final solution (i.e., not setting a 
maximum on the number of intermediate products), and calculating the compositions and costs involved, the case company could get familiar with the model, and grew more confident in its potential.

\subsection{Optimal composition of the potential intermediate products}

In this stage, the composition of the potential intermediate products is determined, based on a fairly simple MILP model (labelled MILP 1) for each of the intermediates $p \in P$. The objective function minimizes the raw material costs:

$$
\operatorname{Min} \sum_{i \in I} f_{i p} c_{i}
$$

where $c_{i}$ is the unit cost for raw material $i$. The quality constraints for the intermediate products are determined according to (1)-(4):

$$
\begin{aligned}
& Q_{p k}^{\min } \leq \sum_{i \in I} f_{i p} Q_{i k} \leq Q_{p k}^{\max } \\
& e^{\frac{Q_{p .9}^{\min }}{1000}} \leq \sum_{i \in I} f_{i p} e^{\frac{Q_{i, 9}}{1000}} \leq e^{\frac{Q_{p, 9}^{\max }}{1000}}, \\
& \sum_{i \in I} f_{i p}=1 .
\end{aligned}
$$$$
\forall k \in\{1, \ldots, 8\}
$$

Constraint (8) deals with the material balance for the intermediate product. For modelling purposes, it was chosen to formulate the quality constraints in (6) in a uniform way, always including a maximum and a minimum. For some of the constraints, we do however not have both. For instance, the DON level (parameter 5) only has a maximum, and the falling number (parameter 8) only has a minimum (as higher falling numbers mean less enzyme activity which is related to less sprout damage). In these cases, the minimum or maximum values are set to zero or infinity, respectively.

For quality parameter 2 , the water absorption ability, there is another constraint in addition to the one in (6). If a certain intermediate product is already in use, the water absorption ability cannot change too much. This leads to a constraint relating $Q_{p, 2}$ to its current value $Q_{p, 2}^{0}$ :

$$
\left(1-\frac{l}{100}\right) Q_{p, 2}^{0} \leq \sum_{i \in I} f_{i p} Q_{i, 2} \leq\left(1+\frac{u}{100}\right) Q_{p, 2}^{0},
$$

where $l$ and $u$ are percentages that the product can decrease or increase in water absorption.

To be able to set constraints on the fractions $f_{i p}$, we introduce a set of indicator variables. These are necessary to distinguish between raw materials that are used and raw materials that are not used (only the first are constrained):

$$
\delta_{i p}=\left\{\begin{array}{lc}
1, & \text { if raw material } i \text { is used in intermediate product } p, \\
0, & \text { otherwise. }
\end{array}\right.
$$

Now, we can define minimum $\left(f_{i p}^{\min }\right)$ and maximum $\left(f_{i p}^{\max }\right)$ fractions in case a raw material is used in the intermediate products:

$$
\delta_{i p} f_{i p}^{\min } \leq f_{i p} \leq \delta_{i p} f_{i p}^{\max } \quad \forall i \in I .
$$

Finally, certain customers require that their product contains at least a certain percentage $\theta_{p}$ of a certain type of raw material $(R \subset I)$, which we therefore also require from a subset of the potential intermediate products $P_{S} \subset P$ : 


$$
\sum_{i \in R \subset I} f_{i p} \geq \theta_{p}
$$

Solving model MILP 1, as defined by equations (5)-(12), for each of the potential intermediate products will result in the optimal composition, in terms of minimal material costs.

\subsection{Selection of intermediate products}

In the third stage, the resulting compositions of potential intermediate products are used in a selection process to determine which intermediate products will eventually be used in the creation of the final products. Again, this results in an MILP model (MILP 2). This time the costs to minimize consist of two parts, mixing operation costs and material costs:

$$
\operatorname{Min} \sum_{j \in J}\left(\beta_{j} D_{j} c_{\beta}+\sum_{p \in P} f_{p j} c_{p} D_{j}\right),
$$

where $c_{\beta}$ the mixing cost per unit of final product, which is multiplied by the demand for product $j$, $D_{j}$. Whether or not mixing is necessary for a certain product is included through:

$$
\beta_{j}=\left\{\begin{array}{cc}
1, & \text { if mixing is necessary for product } j, \\
0, & \text { otherwise. }
\end{array}\right.
$$

Material cost is based on $f_{p j}$, the fractions of intermediate products $p$ in final product $j$, and $c_{p}$ is the material cost for intermediate product $p$, which are both outcomes of MILP 1 .

Similar to MILP 1, we again include quality constraints for all final products, based on the quality parameter values of the intermediate products $Q_{p k}$, and the blending fractions $f_{p j}$.

$$
\begin{array}{ll}
Q_{j k}^{\min } \leq \sum_{p \in P} f_{p j} Q_{p k} \leq Q_{j k}^{\max } & \forall k \in\{1, \ldots, 8\}, \forall j \in J, \\
e^{\frac{Q_{j, 9}^{\min }}{1000}} \leq \sum_{p \in P} f_{p j} e^{\frac{Q_{p, 9}}{1000}} \leq e^{\frac{Q_{j, 9}^{\max }}{1000}} & \forall j \in J, \\
\sum_{p \in P} f_{p j}=s_{j} & \forall j \in J .
\end{array}
$$

As can be seen, the fractions do not sum to 1 in this model, but to a parameter $s_{j}$ which is often still 1, but sometimes has a value smaller than 1 (typically between 0.85 and 1). This is the case, because some final products have additional ingredients that are added in the final stages. This is left out of the model presented in this paper, because it does not affect the final quality of the product.

The additional constraint for the water absorption ability changes is similar to its MILP 1 version:

$$
\left(1-\frac{l}{100}\right) Q_{j, 2}^{0} \leq \sum_{p \in P} f_{p j} Q_{p, 2} \leq\left(1+\frac{u}{100}\right) Q_{j, 2}^{0} \quad \forall j \in J .
$$

To make sure the customer requirement of at least a certain percentage of a certain type of raw material $(R \subset I)$ is continued from the intermediate products, we use:

$$
\sum_{p \in P_{s} \subset P}\left(\sum_{i \in I_{s} \subset I} f_{i p}^{(1)}\right) f_{p j} \geq \theta_{j} \quad \forall j \in J_{s} \subset J .
$$

For reasons of operational simplicity, the management of the company would like to use a maximum number of intermediate products per final product, which can be formulated as: 


$$
\sum_{p \in P} \gamma_{p j} \leq M
$$$$
\forall j \in J
$$

where

$$
\gamma_{p j}=\left\{\begin{array}{lc}
1, & \text { if intermediate product } \mathrm{p} \text { is used in product } j, \\
0, & \text { otherwise }
\end{array}\right.
$$

and $M$ is the maximum number of intermediate products used per final product. To make sure the binary variables $\gamma_{p j}$ have the right values, we use the following constraint:

$$
f_{p j} \leq \gamma_{p j} \quad \forall p \in P, \forall j \in J .
$$

As the mixing stage has a limited capacity, the following constraint assures that we do not use more than the existing mixing capacity $L$.

$$
\sum_{j \in J} \beta_{j} D_{j} \leq L
$$

Again, an additional constraint is introduced to set the binary variable used:

$$
\sum_{p \in P} \gamma_{p j}-1 \leq M \cdot \beta_{j} \quad \forall j \in J .
$$

One of the most essential constraints in this model is the maximum number of intermediate products we allow the model to choose. This is formulated as:

$$
\sum_{p \in P} \lambda_{p} \leq P^{*}
$$

where the binary variable $\lambda_{p}$ is defined as:

$$
\lambda_{p}=\left\{\begin{array}{lc}
1, & \text { if intermediate product } \mathrm{p} \text { is used, } \\
0, & \text { otherwise, }
\end{array}\right.
$$

and this gets the correct value using the following constraint:

$$
\sum_{j \in J} f_{p j} \leq M \cdot \lambda_{p} \quad \forall p \in P .
$$

Furthermore, there are additional constraints due to the fact that some of the intermediates are not allowed to be used in some final products, due to certain characteristics. This leads to a distinction between products that are used to produce bread and products that are not. For this, we define a binary parameter as follows:

$$
\alpha_{p j}=\left\{\begin{array}{cc}
1, & \text { if intermediate } p \text { can be used in product } j, \\
0, & \text { otherwise },
\end{array}\right.
$$

which leads to the following constraints:

$$
f_{p j} \leq \alpha_{p j} \quad \forall p \in P, \forall j \in J .
$$

Solving model MILP 2, as defined by equations (13)-(29), will result in the selection of a preset number of intermediate products. This stage can be repeated for different numbers of resulting intermediate products.

\section{Results}

Due to confidentiality requirements, the actual costs are not reported in this section. We do however report the cost differences between various scenarios for the number of intermediate products by 
using indexed results. To give an indication of the performance of the model, we can compare the solution with 15 intermediate products with the current situation at the company, which would lead to a cost decrease of $0.6 \%$. Although this is fairly limited in terms of the relative difference, the absolute difference still makes the use of the model interesting, as food manufacturers often work with fairly low profit margins. More importantly, the model has proven valuable in tactical and strategic discussions, which we will come back to in the managerial implications in Section 5.3.

The model was implemented in ILOG's OPL software and solved with CPLEX. The calculation times for the results presented in this section range from a few seconds to a few minutes.

\subsection{Number of intermediate products}

The main aspect the company was interested in was an overview of how changing the number of intermediate products $\left(P^{*}\right)$ would affect costs, and how large the cost increases were that they expected to see with a decrease in the number of intermediate products (assuming this would require more flexible, higher quality, and more expensive raw materials). This decrease was expected to simplify the operations and the efficiency of the milling process. We set the cost (including raw material cost and mixing cost) found for the solution with 15 intermediate products as 100 , as this is the current number of intermediate products. This presentation format facilitates the understanding of what happens to the costs when changing the number of intermediate products.

\section{[FIGURE 3 ABOUT HERE]}

Figure 3 shows that decreasing the number of intermediate products below the current number of 15 does increase costs, in what seems to be an exponential curve. Based on these results, it seems that the number of intermediate products can be decreased down to 10 while only marginally increasing costs. Reducing the number of intermediate products further has a larger impact on the costs. It seems that more expensive intermediate products are necessary to provide the flexibility to mix the required end products. This is reflected in e.g. the set of intermediates used in case of 8 intermediates that is not totally part of the larger sets of intermediates. It should be noted that for numbers of intermediates below 7 , it is not possible anymore to design a set of intermediates that can be mixed to create all required final products, and therefore no results can be generated for these scenarios.

\subsection{Choosing the decoupling point: deliver-from-stock or mix-to-order}

As both final products and flexible (still to be mixed) products are considered for intermediate products, deciding on which ones to use also decides on the decoupling point for all final products. Essentially, the choice is between deliver-from-stock (when final products are selected as intermediates) and mix-to-order (when flexible recipes are stored and subsequently mixed when customer orders arrive). It should be noted that in case a final product is selected as an intermediate, it can then also be used as an ingredient in mix-to-order products.

In the scenario with 15 intermediate products, we can see that 9 existing final products are selected as intermediates, in addition to 6 flexible products. Furthermore, due to overlapping intervals in quality specifications, some of the selected final products have such specifications that they can also be substituted for other final products, thereby actually increasing the number of deliver-from-stock products from 9 to 14, and further reducing mixing operations.

\section{[FIGURE 4 ABOUT HERE]}

Figure 4 illustrates how the split between flexible and final products changes for fewer or more intermediate products. For final products, a distinction is made between final products that are also used as ingredients for other final products (multi-use), and final products that are not (single-use). We can see that, for fewer intermediate products, all of them are used in mixing other final 
products. When the number of intermediate products increases, there is an increasing share of single-use final products. Furthermore, these results illustrate that the final products that are chosen to be stored as intermediates often have quality specifications that make them useful as ingredients in other products. Only if we allow a significant increase in the number of intermediate products, we will see a decrease in the use of intermediates as ingredients for other products.

Comparing the final products that still have to be mixed with the ones that can now be delivered directly from storage, we see that the products that can be delivered without mixing have -on average- a larger demand volume; out of the top-10 products in terms of demand volume, only 4 products still need to be mixed. Based on a typical month of demand data, Table 1 illustrates the difference in demand volumes. Like in the previous section, the data is again indexed, here by setting the average demand per product in this specific month to 100.

\section{[TABLE 1 ABOUT HERE]}

These results correspond to what one might expect based on decoupling point theory: for products with large demand volumes earlier specification seems sensible. Although it was not considered in our model, the predictability of demand for these products might also be higher, which would also argue for an upstream effect on the decoupling point (Van Donk, 2001).

To be able to use the 6 flexible intermediate products (from the scenario with 15 products) to mix the remaining final products, it would be sensible to choose a set of products that cover a wide quality spectrum. To see how the model results relate to this, we analyzed the quality specifications of the 6 selected products, in relation to the 30 potential intermediate products designed by the quality management department of the case company.

As it is not practical to illustrate all 9 dimensions of the quality spectrum, we illustrate the results using the parameters protein content and bread volume (quality parameter 1 and 9 as described in Section 3.2). Figure 5 shows the values for these parameters for all 30 flexible intermediate products, and highlights the 6 selected products. Again, results were indexed; here by setting the average value for the specific parameter to 100 . We can see that the model indeed selected a set of products that cover the quality spectrum to a large extent. It is worth noting that the six products shown have fairly similar values for the other quality parameters, with the exception of the 3 products clusters around a protein index of 105-110. These products differ significantly in terms of the fifth quality parameter: DON level, which also explains why these three products are quite close to each other in this illustration. Further outcomes for other quality parameters are not presented in this paper, but show similar results.

\section{[FIGURE 5 ABOUT HERE]}

\subsection{Managerial implications}

Based on the results presented in Sections 5.1 and 5.2, the research team and management along with people directly involved, have discussed the approach and outcomes of the model. All agreed that well-informed decisions can be made regarding the number of intermediate products, their compositions, and whether they are made to stock or mixed to order. The company acknowledges the value of the outcomes and their validity. The outcomes of the model confirmed the viability of the company's policy of having as little as possible intermediates, while keeping all costs low as well. This also confirmed the market approach of the company to provide a broad range of customer-specific products and to further increase that range in the future. The model illustrated that this would not have to be matched by an increase in intermediate products. Further, the model's outcomes facilitate discussion on the overall production strategy, investments in processing equipment, intermediate storage silos and mixing capacity, based on facts and figures. Although the results are consistent with the current way of working and are accepted by the company, the detailed outcomes of the model are not used yet, for reasons of product integrity. Moreover, running 
the mill involves daily small changes in the recipes to accommodate for small differences in lots of ingredients. Below we discuss some of the above issues in some more depth.

When making the final decision on the number of intermediate products, the amount of intermediate storage tanks and the possible cost of investment in additional intermediate storage tanks could play a deciding role. For example, additional cost savings on raw materials and operating costs might not be high enough to warrant the investment in additional storage tanks.

Also, factors like required customer lead times could influence the final decision, as it is very well possible that certain final products have to be available on the intermediate storage level (delivered from stock) to ensure immediate delivery or packaging without further processing operations. If such preferred customer treatment should exist, this could easily be formulated in the model to see if and how that would change the selection of the remaining intermediate products.

By using a limited number of flexible intermediate products ( 6 in the scenario discussed in Section 5.2), postponement of specification for a large range of final products (here 31) can be achieved. This downstream effect in terms of the decoupling point can be used to control demand uncertainty, both in terms of which products will be ordered and when they will be ordered (Yang et al., 2004a).

Finally, it is worth noting that, following Graman and Magazine (2006), successful implementations of postponement not only build on selecting the right products, but also on how the results are translated into other organizational functions such as operations scheduling, where different types of products might have to be dealt with in different ways. Graman and Magazine also stress that care should be taken related to possible changes in product integrity. As our case study concerns an application in the food industry, this relates to food quality and safety, which is indeed something that should be considered very carefully.

All in all, based on the experiences gathered in our case study, we are sure that the model can provide a valuable tool to support decision making at several levels. As with all models it will not replace decision making.

\section{Conclusion and discussion}

In this paper, we develop a method to design intermediate products in a two-stage food production process, taking into account a wide variety of quality-related attributes. The method consists of a two-stage mathematical programming model that aims to find a balance between material cost and operating cost. The model is developed and applied in a case study concerning a medium-sized flour manufacturer.

The results show that the number of intermediate products can be reduced, simplifying operations and increasing efficiency of the milling process, while only leading to a marginal cost increase. Several scenarios have been studied for operational costs and raw material availability to validate the results, also illustrating the usefulness of the model as a decision support tool for the make or mix to order decision. It also shows that the costs increase if the number of intermediate products decreases, although cost changes are marginal above a certain number of intermediates. The model is designed to be used for what-if analysis and should be solved on a regular basis, to make sure the current market environment (including developments in the raw material supply and the customer demand) is reflected in the composition of the intermediate products. Even more important is that the results and scenarios of the model can be used to discuss and develop the production strategy, explore consequences in terms of investments needed, etc., based on objective figures and comparisons, in addition to experience, traditions and tacit knowledge.

Although the paper largely describes one single case study, the model developed can easily be adapted for other food production systems, as many of these can be seen as two-stage production systems with intermediate storage and a divergent product flow. Furthermore, a lot of the quality attributes modelled in the case study are typical for the food industry, which suggests numerous possible applications of the approach and the model.

Scientifically, this paper contributes in several ways. We incorporate the design of intermediate products to Decoupling Point theory, whereas such intermediates are normally given. We help in 
developing quantitative, but also integral decision tools to that body of knowledge. It is clear that the model can help managers to make better-informed decisions regarding what products to store as intermediates.

Future research could follow a number of directions. One possible way is to incorporate more elements into our quantitative model: constraints relating to raw material usage or availability, or capacity limitations such as storage or batch sizes. Related to these additional constraints, it might be beneficial to create an integrated model instead of the decomposed approach presented in this paper. This would allow for better ways to include e.g. constraints on raw material usage. Also, in an integrated model, we would not need to limit the list of potential flexible intermediate products, as we could let the model design these, based on the available raw materials and the required final products. An integrated model would be more complex from a computational viewpoint, but methods that could be used to solve such a problem in an efficient way are available in the literature (e.g. Benders, 1962). However, for the approach presented in this paper, we aimed at supporting the current organizational decision-making process and did not pursue an integrated modelling approach. Also the results of the current model are therefore accepted more easily. Finally, more research is also needed to further explore how a tool like the one developed here, should and can be used in the organisational context. Here, we need to decide how often the models have to be solved with updated information, and identify which decisions (be they strategic, tactical or operational) the tool could also support, next to the issues discussed in this paper.

\section{Acknowledgements}

The authors would like to thank the company involved for their willingness to participate in this research and for their suggestions and remarks during discussion sessions. Also, the first author would like to acknowledge support from a H.C. Ørsted postdoctoral fellowship from the Technical University of Denmark.

\section{References}

Abukhader, S.M., Jonson, G., 2007. Postponement strategies in food supply chains - an empirical investigation. International Journal of Logistics Systems and Management, 3(2), 215-234.

Akkerman, R., Van Donk, D.P., Gaalman, G., 2007. The influence of capacity- and timeconstrained intermediate storage in two-stage food production systems. International Journal of Production Research, 45(13), 2955-2973.

Ashayeri, J., Van Eijs, A.G.M., Nederstigt, P., 1994. Blending modelling in a process manufacturing: A case study. European Journal of Operational Research, 72(3), 460-468.

Benders, J.F., 1962. Partitioning procedures for solving mixed-variables programming problems. Numerische Mathematik, 4, 238-252.

Boone, C.A., Craighead, C.W., Hanna, J.B., 2007. Postponement: an evolving supply chain concept. International Journal of Physical Distribution \& Logistics Management, 37(8), 594-611.

Caux, C., David, F., Pierreval, H., 2006. Implementation of delayed differentiation in batch process industries: a standardization problem. International Journal of Production Research, 44(16), 32433255.

Dobson, P.W., Clarke, R., Davies, S., Waterson, M., 2001. Buyer power and its impact on competition in the food retail distribution sector of the European Union. Journal of Industry, Competition and Trade, 1(3), 247-281.

Forza, C., Salvador, F., Trentin, A., 2008, Form postponement effects on operational performance: a typological theory, International Journal of Operations \& Production Management, 28(11), 10671094.

Fransoo, J.C., Rutten, W.G.M.M., 1994. A typology of production control situations in process industries. International Journal of Operations \& Production Management, 14(12), 47-57. 
Graman, G.A., Magazine, M.J., 2006. Implementation issues influencing the decision to adopt postponement. International Journal of Operations \& Production Management, 26(10), 1068-1083. Griffith, C.J., 2006, Food safety: Where from and where to? British Food Journal, 108(1), 6-15.

Hoekstra, S., Romme, J.H.J.M. (editors), 1992. Integral logistic structures: Developing customeroriented goods flows. London: McGraw-Hill.

Kerkkänen, A., 2007. Determining semi-finished products to be stocked when changing the MTSMTO policy: Case of a steel mill. International Journal of Production Economics, 108(1-2), 111118.

Lyon, P., Milne, R.J., Orzell, R., Rice, R. 2001, Matching assets with demand in supply-chain management at IBM Microelectronics, Interfaces, 31(1), 108-124.

Olhager, J., 2003. Strategic positioning of the order penetration point. International Journal of Production Economics, 85(3), 319-329.

Rajaram, K., Jaikumar, R., Behlau, F., Van Esch, F., Heynen, C., Kaiser, R., Kuttner, A., Van de Wege, I., 1999. Robust process control at Cerestar's refineries. Interfaces, 29(1), 30-48.

Rutten, W.G.M.M., 1995. The use of recipe flexibility in production planning and inventory control, Ph.D. Thesis, Eindhoven University of Technology, The Netherlands.

Rutten, W.G.M.M., Bertrand, J.W.M., 1998. Balancing stocks, flexible recipe costs and high service level requirements in a batch process industry: A study of a small scale model. European Journal of Operational Research, 110(3), 626-642.

Soman, C.A., Van Donk, D.P., Gaalman, G., 2004. Combined make-to-order and make-to-stock in a food production system. International Journal of Production Economics, 90(2), 223-235.

Van Donk, D.P., 2001. Make to stock or make to order: The decoupling point in the food processing industries. International Journal of Production Economics, 69(3), 297-306.

Van Donk, D.P., Akkerman, R., Van der Vaart, J.T., 2008. Opportunities and realities of supply chain integration: The case of food manufacturers. British Food Journal, 110(2), 218-235.

Van Hoek, R.I., 1999. Postponement and the reconfiguration challenge for food supply chains. Supply Chain Management: An International Journal, 4(1), 18-34.

Van Hoek, R.I., 2001. The rediscovery of postponement: a literature review and directions for research. Journal of Operations Management, 19(2), 161-184.

Wanders, H.L.T., Gaalman, G.J.C., Sierksma, G., 2004. The composition of semi-finished inventories at a solid board plant. European Journal of Operational Research, 155(1), 96-111.

Yang, B., Burns, N.D., Backhouse, C.J., 2004a. Management of uncertainty through postponement. International Journal of Production Research, 42(6), 1049-1064.

Yang, B., Burns, N.D., Backhouse, C.J., 2004b, Postponement: a review and an integrated framework. International Journal of Operations \& Production Management, 24(5), 468-487. 


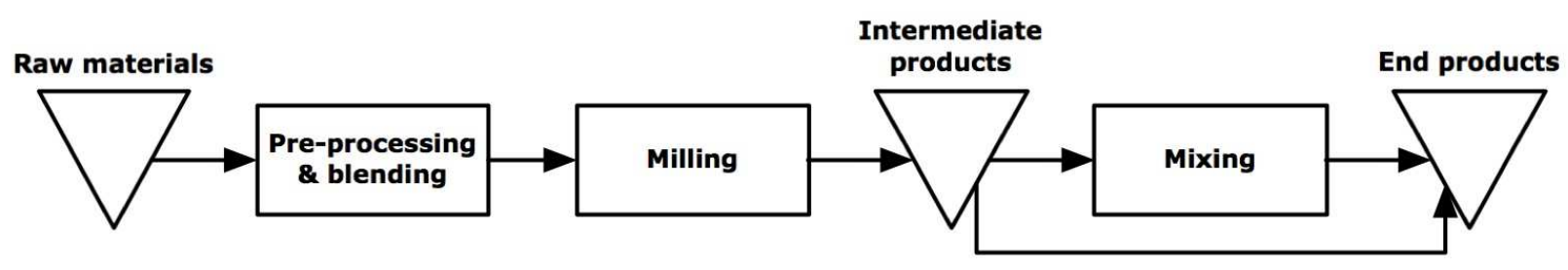

Figure 1 - Outline of the production process of the flour mill. 


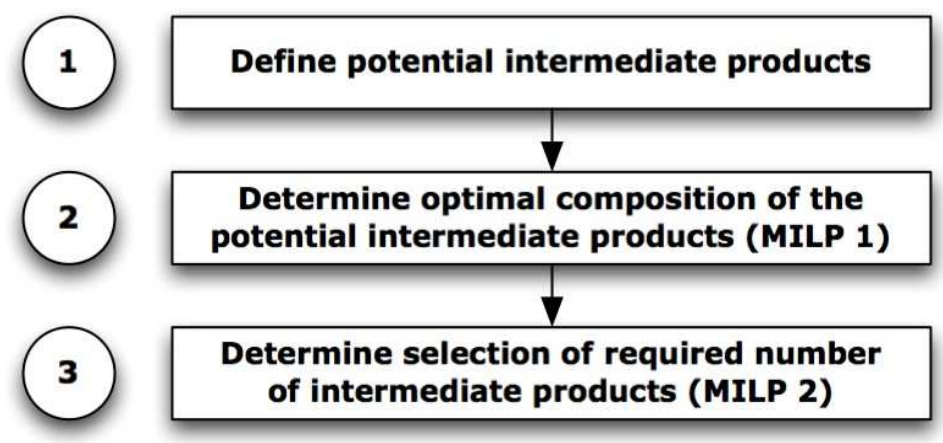

Figure 2 - Schematic representation of the solution approach. 


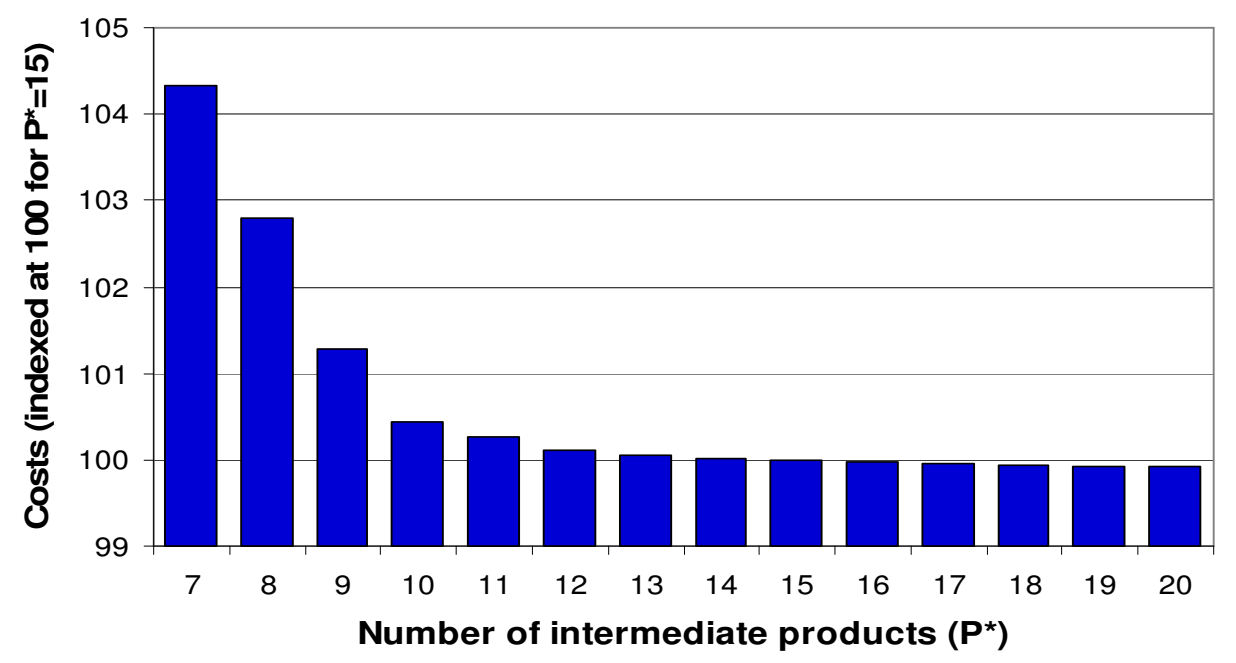

Figure 3 -Cost results (indexed) for several scenarios for the number of intermediate products. 


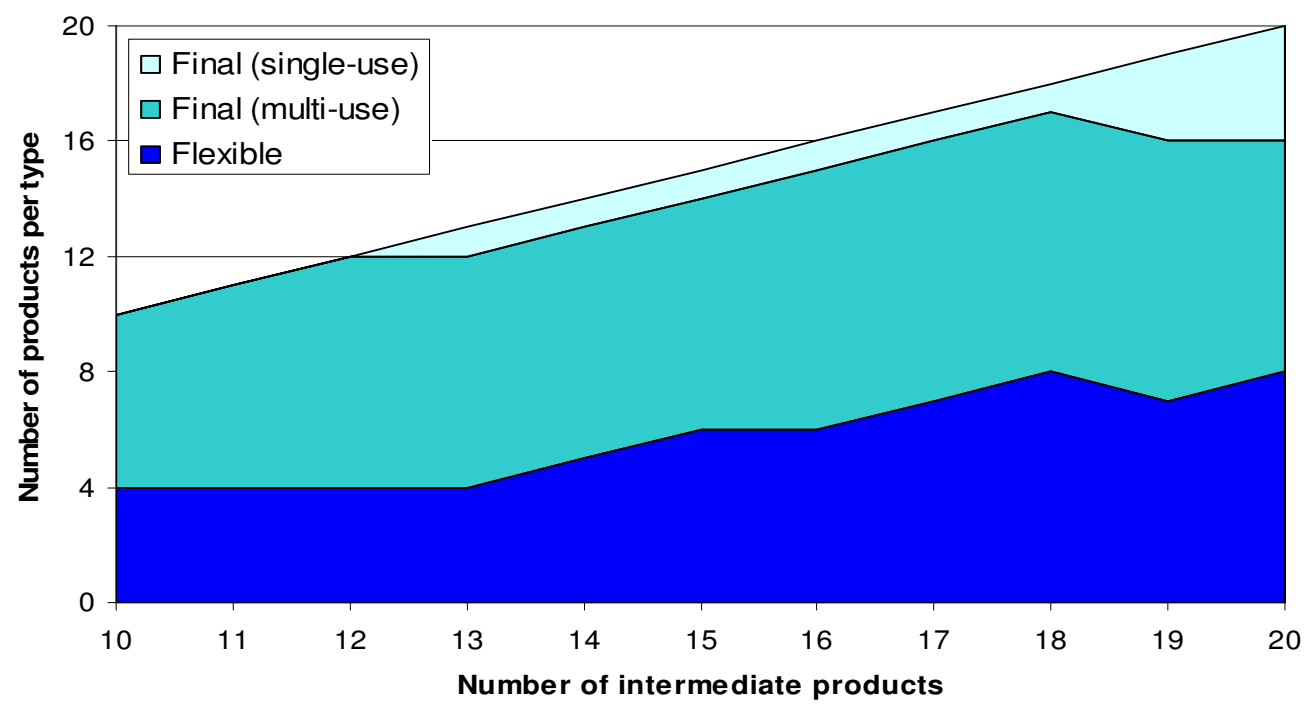

Figure 4 - Split between flexible and final products for several scenarios for the number of intermediate products. 


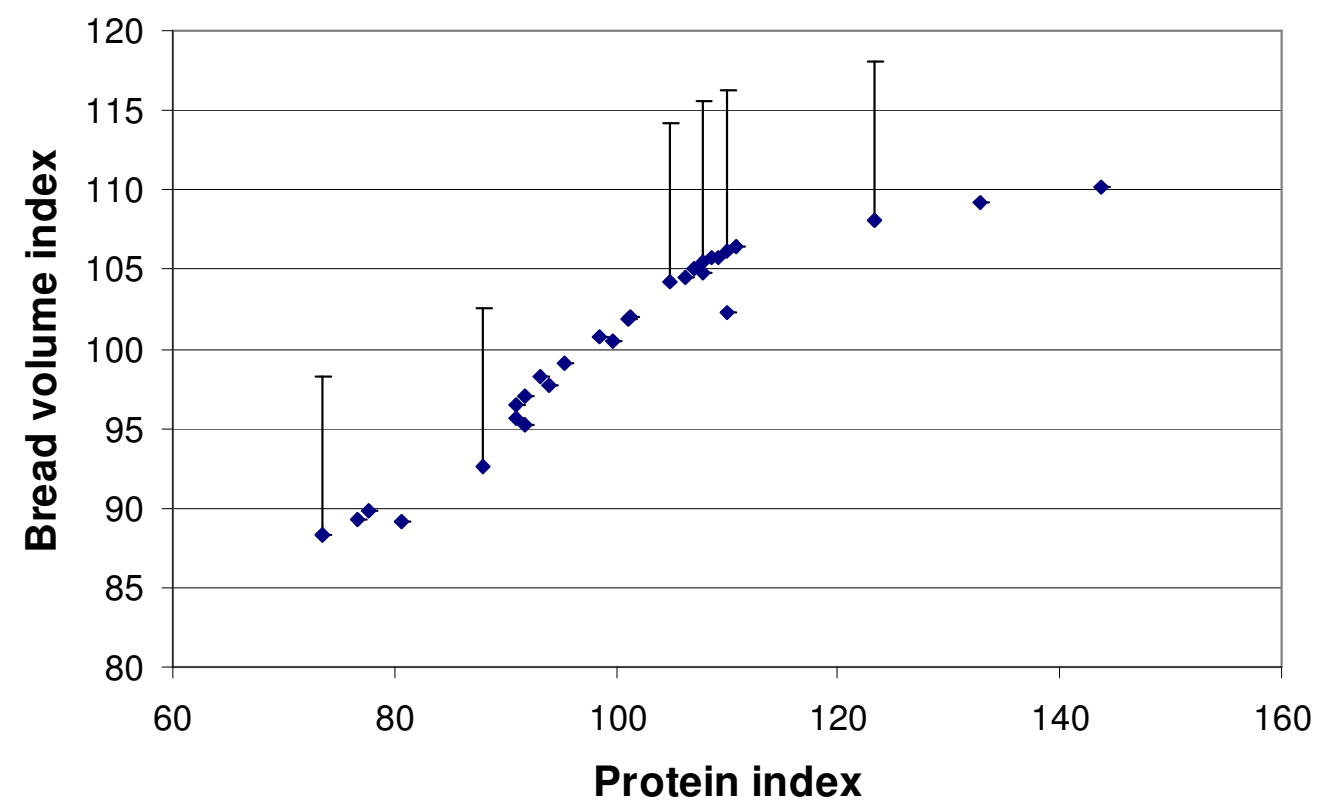

Figure 5 - Illustration of the quality parameter range covered by the six selected flexible intermediate products. 
Table 1 - Comparison of final product demand volumes (indexed) for different products.

\begin{tabular}{lccc}
\hline Product type & $\begin{array}{c}\text { Number of } \\
\text { products }\end{array}$ & $\begin{array}{c}\text { Average demand } \\
\text { volume (+ st. dev. })\end{array}$ & $\begin{array}{c}\text { Share of } \\
\text { demand }\end{array}$ \\
\hline Deliver-from-stock & 14 & $191.7(194.1)$ & $60 \%$ \\
Mix-to-order & 31 & $58.6(96.1)$ & $40 \%$ \\
\hline Total/average & 45 & $100(146.0)$ & $100 \%$ \\
\hline
\end{tabular}

